\title{
478.
}

\section{ON THE GEODESIC LINES ON AN ELLIPSOID.}

[From the Memoirs of the Royal Astronomical Society, vol. xxxix. (1872), pp. 31-553. Read January 13, 1871.]

THE fundamental equations, in regard to the geodesic lines on an ellipsoid, were established by Jacobi, viz., representing by $a, b, c$, the squares of the semiaxes, that is, taking the ellipsoid to be

$$
\frac{x^{2}}{a}+\frac{y^{2}}{b}+\frac{z^{2}}{c}=1
$$

(where $a>b>c$ ), if we introduce the elliptic coordinates $h, k$, and write

or, what is the same thing,

$$
\begin{aligned}
& \frac{x^{2}}{a+h}+\frac{y^{2}}{b+h}+\frac{z^{2}}{c+h}=1, \\
& \frac{x^{2}}{a+k}+\frac{y^{2}}{b+k}+\frac{z^{2}}{c+k}=1,
\end{aligned}
$$

$$
\begin{aligned}
& x^{2}=\frac{a(a+h)(a+k)}{(a-b)(a-c)} \\
& y^{2}=\frac{b(b+h)(b+k)}{(b-c)(b-a)} \\
& z^{2}=\frac{c(c+h)(c+k)}{(c-a)(c-b)}
\end{aligned}
$$

then, if $\beta$ be an arbitrary constant, the differential equation of a geodesic line is

(1) const. $=\int d h \sqrt{\frac{h}{(a+h)(b+h)(c+h)(\beta+h)}}+\int d k \sqrt{\frac{h}{(a+k)(b+k)(c+k)(\beta+k)}}$, 
and the expression for the length of any are of the curve is given by

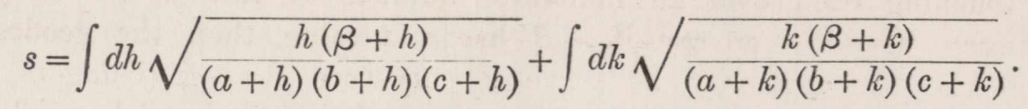

I propose in the present Memoir to develope the theory to the extent of showing how we can, by means of the first of these equations, explain the course of the geodesic lines; and for given numerical values of $a, b, c$, calculate, construct, and exhibit in a drawing the course of these lines: I attend more particularly to the series of geodesic lines through an umbilicus (which lines pass also through the opposite umbilicus), and to the case where the semiaxes are connected by the equation $a c-b^{2}=0$, a relation which simplifies the formulæ.

\section{General Considerations as to the Course of the Lines.}

1. It will be observed that $h$ and $k$ enter into the formulæ symmetrically: it will be convenient to distinguish between these coordinates by considering $h$ as extending between the values $-a,-b$; and $k$ as extending between the values $-b,-c$. Thus:

$h=$ const. denotes a curve of curvature of the one kind, viz.:

$h=-a$, the principal section $A B A^{\prime}$ (or major-mean section), $h=-b$, the curves $U U^{\prime}$ and $U^{\prime \prime} U^{\prime \prime \prime}$ (or portions of the umbilicar section $A C A^{\prime} C^{\prime}$ ); similarly,

$k=$ const. denotes a curve of curvature of the other kind, viz. :

$k=-c$, the principal section $C B C^{\prime}$ (or minor-mean section), $k=-b$, the curves $U U^{\prime \prime \prime}$ and $U^{\prime} U^{\prime \prime}$ (remaining portions of the umbilicar section $A C A^{\prime} C^{\prime}$ ).

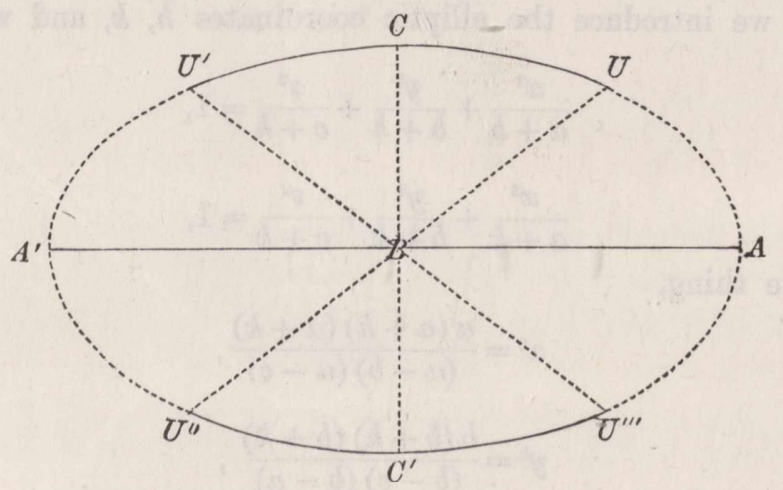

2. To any given (admissible) values of $h, k$ there correspond eight points, situate in the eight octants of the surface respectively; but, unless the contrary is expressed, it is assumed that the coordinates $x, y, z$, are positive, and that the point is situate in the octant $A B C$.

3. The constant $\beta$ may have any value from $+a$ to $+c$; viz., if it has a value between $a$ and $b$, or say, if $-\beta$ has an $h$-value, then the geodesic lines wholly 
between the two ovals of the curve of curvature $h=-\beta$. (being in general an indefinite undulating curve touching each oval an indefinite number of times). Similarly, if $\beta$ has any value between $b$ and $c$, or say, if $-\beta$ has a $k$-value, then the geodesic line lies wholly between the two ovals of the curve of curvature $k=-\beta$ (being in general an indefinite undulating curve touching each oval an indefinite number of times). The intermediate case is when $\beta=b$, or say when $-\beta$ has the umbilicar value: here the geodesic line is in general an indefinite undulating curve passing an infinite number of times through the opposite umbilici $U, U^{\prime \prime}$, or $U^{\prime}, U^{\prime \prime \prime}$; to fix the ideas, say through $U, U^{\prime \prime}$.

\section{Lines through an Umbilicus.}

4. I attend in particular to the last-mentioned case, and thus write $\beta=b$. We may in the formula (1) fix at pleasure a limit of each integral; and writing for convenience

the equation (1) becomes

$$
\begin{aligned}
& \Pi(h)=\int_{-a}^{h} \frac{-d h}{b+h} \sqrt{\frac{h}{(a+h)(c+h)}}, \\
& \Psi(k)=\int_{k}^{-e} \frac{d k}{b+k} \sqrt{\frac{k}{(a+k)(c+k)},}
\end{aligned}
$$

$$
\text { Const. }=\Pi(h)+\Psi(k) \text {. }
$$

5. It is to be observed, in regard to these integrals, that writing $h=-a+u$, we have

which, for $u$ small, is

$$
\Pi(h)=\int_{0}^{u} \frac{d u}{a-b-u} \sqrt{\frac{a-u}{u(a-c-u)}},
$$

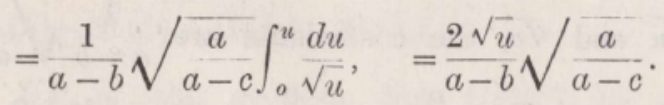

By the assistance of this formula the value of the integral may be calculated by quadratures; viz., the formula gives the integral for any small value of $u$, and we can then proceed by the method of quadratures. The integral becomes infinite for $h=-b$ : suppose that we have by quadratures calculated it up to $h=-b-m$ ( $m$ small), then to calculate it up to any value $-b-m+u$ nearer to $-b$, we have

$$
\begin{aligned}
\Pi(h) & =\Pi(-b-m)+\int_{0}^{u} \frac{d u}{m-u} \sqrt{\frac{b+m-u}{(a-b-m+u)(b-c+m-u)}} \\
& =\Pi(-b-m)+\sqrt{\frac{b}{(a-b)(b-c)} \int_{o}^{u} \frac{d u}{m-u}} \\
& =\Pi(-b-m)-\sqrt{\frac{b}{(a-b)(b-c)}} \log \left(1-\frac{u}{m}\right),{ }^{(1)}
\end{aligned}
$$

where the second term is positive, and the value thus increases slowly with $u$, becoming as it should do $=\infty$ for $u=m$ or $h=-b$.

${ }^{1}$ Except when the contrary is stated, the symbol " $\log$ " denotes throughout the hyperbolic logarithm. 
6. Similarly in the second integral writing $k=-c-v$, we have

which, for $v$ small, is

$$
\Psi(k)=\int_{0}^{v} \frac{d v}{b-c-v} \sqrt{\frac{c+v}{(a-c-v) v}},
$$

$$
=\frac{1}{b-c} \sqrt{\frac{c}{a-c}} \int \frac{d v}{\sqrt{v}}, \quad=\frac{2 \sqrt{v}}{b-c} \sqrt{\frac{c}{a-c}},
$$

which is of the like assistance in regard to the calculation by quadratures. And if we have by quadratures calculated the integral up to $h=-b+n$ ( $n$ small), then, to calculate it up to any value $-b+n-v$ nearer to $-b$, we have

$$
\begin{aligned}
\Psi(k) & =\Psi(-b+n)+\int_{0}^{v} \frac{d v}{n-v} \sqrt{\frac{b-n+v}{(a-b+n-v)(b-c-n+v)}} \\
& =\Psi(-b+n)+\sqrt{\frac{b}{(a-b)(b-c)}} \int_{0}^{v} \frac{d v}{n-v} \\
& =\Psi(-b+n)-\sqrt{\frac{b}{(a-b)(b-c)}} \log \left(1-\frac{v}{n}\right),
\end{aligned}
$$

where the second term is positive, and the value thus increases slowly with $v$, becoming as it should do $=\infty$ for $v=n$, or $k=-b$.

7. It may be remarked that in $\Pi(h)$ and $\Psi(k)$ respectively the coefficient of the logarithmic term has in each case the same value $=\sqrt{\frac{b}{(a-b)(b-c)}}$. As regards the initial terms $\sqrt{u}$ and $\sqrt{v}$, the coefficients are $\frac{1}{a-b} \sqrt{\frac{a}{a-c}}$ and $\frac{1}{b-c} \sqrt{\frac{c}{a-c}}$ respectively, which are equal if $\frac{a}{(a-b)^{2}}=\frac{c}{(b-c)^{2}}$, or $a c-b^{2}=0$.

8. We may consider the two geodesic lines $\Pi(h) \pm \Psi(k)=$ const.; suppose that these each of them pass through the point $P$, coordinates $\left(h_{0}, k_{0}\right)$ in the $A B C$ octant of the ellipsoid; then for one of them we have $\Pi(h)-\Psi(k)=\Pi\left(h_{0}\right)-\Psi\left(k_{0}\right)$, and for the other of them we have $\Pi(h)+\Psi(k)=\Pi\left(h_{0}\right)+\Psi\left(k_{0}\right)$ : I attend first to the former of these, say $\Pi(h)-\Psi(k)=C$ (where $C$ is $\left.=\Pi\left(h_{0}\right)-\Psi\left(k_{0}\right)\right)$; and I say that this denotes the curve $U P U^{\prime \prime}$. In fact, by reason of the equation $\Pi(h)$ and $\Psi(k)$ must both increase or both diminish; they both increase as $h$ passes from $h_{0}$ to $-b$, and as $k$ passes from $k_{0}$ to $-b$ : we may have $h=-b+u, k=-b+v$ where $u$ and $v$ are both indefinitely small, the functions $\Pi$ and $\Psi$ being then indefinitely large, but $\Pi-\Psi=C$; and we have thus a series of points nearer and nearer to the umbilicus $U$; that is, we have the portion $P U$ of the curve. Tracing the curve in the opposite direction, or considering $h$ as passing from $h_{0}$ to $-a$, and $k$ as passing from $k_{0}$ to $-c$, then if $C$ be positive, $k$ will attain the value $-c$, before $h$ attains the value $-a$, say that we have simultaneously $h=h_{1}, k=-c$; the equation is $\Pi\left(h_{1}\right)-\Psi(-c)=C$, that is, $\Pi\left(h_{1}\right)=C$; and the geodesic line then arrives at a point $P$, on the arc $C B$ of the minor-mean 
principal section. The function $\Psi$ then changes its sign, viz., considering it as always positive, the equation is now $\Pi(h)+\Psi(k)=C, k$ passing from the value $-c$ towards $-b$, that is, $\Psi(k)$ increasing, and therefore $\Pi(h)$ diminishing, or $h$ passing from $h_{1}$ towards the value $-a$; until at last, say for $k=k_{2}$, we have $h=-a$, that is, $C=\Pi(-a)+\Psi\left(k_{2}\right)$, or $C=\Psi\left(k_{2}\right)$; the geodesic line here arrives at a point $P_{2}$ on the arc $B A^{\prime}$ of the major mean principal section. The function $\Pi$ then changes its sign, viz., $\Pi$ denoting a positive function as before, the equation is $-\Pi(h)+\Psi(k)=C$; $h$ passes from $-a$ towards $-b$, that is $\Pi(h)$ increases, and therefore $\Psi(k)$ must also increase, or $k$ pass from $k_{2}$ towards $-b$ : we have at length $h=-b-u, k=-b+v$, $u$ and $v$ being each indefinitely small; and therefore $\Pi$ and $\Psi$ each indefinitely large (but $-\Pi+\Psi=C$ ); that is, we arrive at the umbilicus $U^{\prime \prime}$, completing the geodesic line $U P U^{\prime \prime}$.

9. If instead of $C=+$ we have $C=-$, everything is similar, but the geodesic line proceeding from $U$ in the direction $U P$ will first cut the arc $B A$ of the major mean section at a point $P_{1}$; then the arc $B C^{\prime}$ of the minor mean section at a point $P_{2}$; and, finally, arrive as before at the umbilicus $U^{\prime \prime \prime}$.

10. The intermediate case is when $C=0$, viz., we have here $\Pi(h)-\Psi(k)=0$; the geodesic line here passes from $U$ in the direction $U P$ to $B$ (extremity of the mean axis, $h=-a, k=-c) ; \Pi$ and $\Psi$ then each change their sign, so that, considering them as positive, the equation still is $\Pi(h)-\Psi(k)=0$, and the geodesic line at last arrives at the umbilicus $U^{\prime \prime}$. It will be easily understood how in the like manner $\Pi(h)+\Psi(k)=C$ refers to the line $U^{\prime} P U^{\prime \prime \prime}$.

11. Reverting to the equation $\Pi(h)-\Psi(k)=C$, or as I will now write it

$$
\Pi(h)-\Psi(k)=\Pi\left(h_{0}\right)-\Psi\left(k_{0}\right),
$$

which belongs to the portion $U P$ of the geodesic line $U P U^{\prime \prime}$, we require when $h$ is $=-b-u$, and $k=-b+v$ ( $u$ and $v$ indefinitely small) to know the ratio of the increments $u, v$; this in fact serves to determine the direction at $U$ of the geodesic line through the given point $\left(h_{0}, k_{0}\right)$.

12. For this purpose writing $h=-b-u$, we find

which is

$$
\Pi(h)=\int_{u}^{a-b} \frac{d u}{u} \sqrt{\frac{b+u}{(a-b-u)(b-c+u)}},
$$

$$
\begin{gathered}
=\int_{u}^{a-b} \frac{d u}{u}\left\{\sqrt{\frac{b+u}{(a-b-u)(b-c+u)}}-\sqrt{\frac{b}{(a-b)(b-c)}}\right\} \\
+\sqrt{\frac{b}{(a-b)(b-c)}} \log \frac{a-b}{u},
\end{gathered}
$$

and, when $u$ is indefinitely small, this is

$$
\Pi(h)=\int_{0}^{a-b} \frac{d u}{u}\left\{\sqrt{\frac{b+u}{(a-b-u)(b-c+u)}}-\sqrt{\frac{b}{(a-b)(b-c)}}\right\}+\sqrt{\frac{b}{(a-b)(b-c)}} \log \frac{a-b}{u} .
$$

c. VII. 
Similarly, when $k=-b+v$, where $v$ is indefinitely small

$$
\Psi(k)=\int_{0}^{b-c} \frac{d v}{v}\left\{\sqrt{\frac{b-v}{(a-b+v)(b-c-v)}}-\sqrt{\frac{b}{(a-b)(b-c)}}\right\}+\sqrt{\frac{b}{(a-b)(b-c)}} \log \frac{b-c}{v} .
$$

13. Each of the integrals is of the dimension $-\frac{1}{2}$ in $a, b, c$, and the difference of the integrals may be represented by

we have therefore

$$
M \sqrt{\frac{b}{(a-b)(b-c)}}
$$

where

$$
\Pi(h)-\Psi(k)=\sqrt{\frac{b}{(a-b)(b-c)}}\left\{M+\log \frac{a-b}{b-c} \frac{v}{u}\right\},
$$

$$
\begin{aligned}
\sqrt{\frac{b}{(a-b)(b-c)} M} & =\int_{0}^{a-b} \frac{d u}{u}\left\{\sqrt{\frac{b+u}{(a-b-u)(b-c+u)}}-\sqrt{\left.\frac{b}{(a-b)(b-c)}\right\}}\right. \\
& -\int_{0}^{b-c} \frac{d v}{v}\left\{\sqrt{\frac{b-v}{(a-b+v)(b-c-v)}}-\sqrt{\frac{b}{(a-b)(b-c)}}\right\} .
\end{aligned}
$$

14. Suppose the inferior limits replaced by the indefinitely small positive quantities $\epsilon, \epsilon^{\prime}$ respectively; and for the variable in the second integral write $-u$; then

$$
M=\int_{-(b-c)}^{a-b}\left\{\frac{d u}{u} \sqrt{\frac{b+u}{(a-b-u)(b-c+u)}}-\sqrt{\frac{b}{(a-b)(b-c)}}\right\},
$$

it being understood that the values $u=-\epsilon^{\prime}$ to $u=+\epsilon$ are omitted from the integration: this is

$$
=\int_{-(b-c)}^{a-b} \frac{d u}{u} \sqrt{\frac{b+u}{(a-b-u)(b-c+u)}}-\sqrt{(a-b)(b-c)} \log _{\epsilon} \frac{b-b}{\epsilon^{\prime}}
$$

with the same convention as to the integral; or if $\epsilon^{\prime}=\epsilon$, then

where

$$
M=M^{\prime}-\sqrt{\frac{b}{(a-b)(b-c)} \log _{b-c}^{a-b}}
$$

$$
\sqrt{\frac{b}{(a-b)(b-c)}} M^{\prime}=\int_{-(b-c)}^{a-b} \frac{d u}{u} \sqrt{\frac{b+u}{(a-b-u)(b-c+u)}}=\int_{-a}^{-c} b+h \sqrt{\frac{h}{(a+h)(c+h)}},
$$

the omitted elements being from $u=-\epsilon$ to $u=+\epsilon$; that is (in the language of Cauchy) we take for the integral its principal value. And hence

$$
\Pi(h)-\Psi(k)=\sqrt{\frac{b}{(a-b)(b-c)}\left\{M^{\prime}+\log \frac{v}{u}\right\}} .
$$

15. By what precedes this is $=\Pi\left(h_{0}\right)-\Psi\left(k_{0}\right)$; or if we write simply $(h, k)$ instead of $\left(h_{0}, k_{0}\right)$, that is, consider the geodesic line UP, which is drawn from the 
point $P$, coordinates $(h, k)$, to the umbilicus $U$, the coordinates of a point consecutive to the umbilicus are $-b-u,-b+v$, where $u, v$ are connected by the last-mentioned equation, in which $M^{\prime}$ is a transcendental function depending on $(a, b, c)$ but independent of the particular geodesic line.

16. If for the geodesic line through the point $B$, or say for the $B$-geodesic $\frac{v}{u}=\frac{v_{0}}{u_{0}}$, then $M^{\prime}=-\log \frac{v_{0}}{u_{0}}$, and we have in general

$$
\Pi(h)-\Psi(k)=\sqrt{\frac{b}{(a-b)(b-c)}} \log \frac{v u_{0}}{u v_{0}},
$$

a result which I proceed to further transform as follows:

If $x_{0}, y_{0}, z_{0}$ refer to the umbilicus $U$, then considering first the consecutive point $P$ on the geodesic line (coordinates $-b-u,-b+v$ ) and next the consecutive

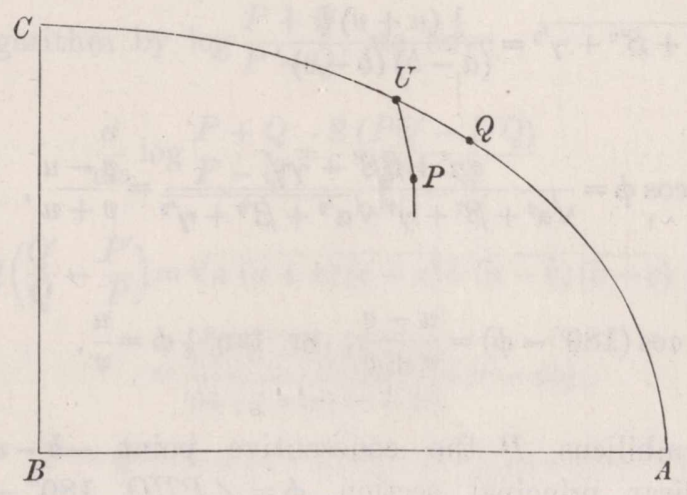

point $Q$ on the umbilicar section, we have for these two points respectively,

$$
\begin{aligned}
& d x_{0}=\frac{\frac{1}{2} \sqrt{a}(v-u)}{\sqrt{(a-b)(a-c)}}, \\
& d y_{0}=\frac{\sqrt{b} \sqrt{u v}}{\sqrt{(a-b)(b-c)}}, \\
& d z_{0}=\frac{\frac{1}{2} \sqrt{c}(u-v)}{\sqrt{(b-c)(a-c)}}, \\
& \delta x_{0}=\frac{\frac{1}{2} \sqrt{a}}{\sqrt{(a-b)(a-c)}}, \\
& \delta y_{0}=0, \quad-\frac{-1}{2} \sqrt{c} \\
& \delta z_{0}=\frac{\sqrt{(b-c)(a-c)}}{\sqrt{(b-c)}}
\end{aligned}
$$


say these are $\alpha, \beta, \gamma$, and $\alpha^{\prime}, \beta^{\prime}, \gamma^{\prime}$; and then

$$
\begin{aligned}
\alpha \alpha^{\prime}+\beta \beta^{\prime}+\gamma \gamma^{\prime} & =\frac{1}{4}\left\{\frac{a}{(a-b)(a-c)}+\frac{c}{(b-c)(a-c)}\right\}(v-u), \\
\alpha^{2}+\beta^{2}+\gamma^{2} & =\left\{\frac{\frac{1}{4}(v-u)}{a-c}\left\{\frac{a}{(a-b}+\frac{c}{b-c}\right\},=\frac{\frac{1}{4} b(v-u)}{(a-b)(b-c)},\right. \\
& \left.=\frac{b}{(a-b)(b-c)} \cdot \frac{c}{(a-c)(a-c)}\right\} \cdot \frac{1}{4}(u+v)^{2}, \\
\alpha^{\prime 2}+\beta^{\prime 2}+\gamma^{\prime 2} & =\frac{b}{(a-b)(b-c)} \cdot \frac{b u v}{(a-b)(b-c)}
\end{aligned}
$$

whence

$$
\sqrt{\alpha^{2}+\beta^{2}+\gamma^{2}} \sqrt{\alpha^{\prime 2}+\beta^{\prime 2}+\gamma^{\prime 2}}=\frac{\frac{1}{4}(u+v) b}{(a-b)(b-c)},
$$

and hence

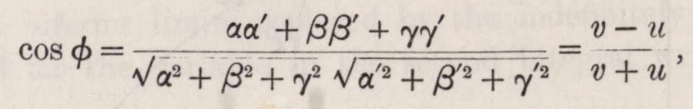

that is,

$$
\cos \left(180^{\circ}-\phi\right)=\frac{u-v}{u+v}, \text { or } \tan ^{2} \frac{1}{2} \phi=\frac{u}{v},
$$

where if $U$ is the umbilicus, $P$ the consecutive point $-b-u,-b+v$, and $U Q$ the element of the umbilicar principal section, $\phi=\angle P U Q, 180^{\circ}-\phi=\angle P U Q^{\prime}$. For the $B$-geodesic we have

$$
2 \log \tan \frac{1}{2} \phi_{0}=\log \frac{u_{0}}{v_{0}}=M^{\prime}
$$

17. The foregoing equation for $\Pi(h)-\Psi(k)$ now becomes

$$
\Pi(h)-\Psi(k)=\sqrt{\frac{b}{(a-b)(b-c)}} \log \frac{\tan ^{2} \frac{1}{2} \phi_{0}}{\tan ^{2} \frac{1}{2} \phi} ;
$$

viz., $\phi_{0}$ is the south azimuth of the $B$-geodesic at the umbilicus, a mere function of $(a, b, c)$ and $\phi$ is the south azimuth at the umbilicus, of the geodesic line under consideration, so that we may consider the geodesic line to be determined by the south azimuth $\phi$ as its parameter. 
Formulce for the case $a c-b^{2}=0$.

18. I annex the following investigation in regard to the case $a c-b^{2}=0$.

We have in general

$$
\begin{aligned}
\frac{1}{\sqrt{b(a-b)(b-c)} \frac{d}{d x} \log } \frac{\sqrt{-x(a-b)(b-c)}+\sqrt{-b(a+x)(c+x)}}{\sqrt{-x(a-b)(b-c)-\sqrt{ }-b(a+x)(c+x)}} \\
=-\frac{1}{b} \frac{1}{\sqrt{x(a+x)(c+x)}} \\
+\frac{1}{b} \frac{1}{b+x} \sqrt{\frac{x}{(a+x)(c+x)}} \\
+\frac{1}{b x+a c} \sqrt{\frac{x}{(a+x)(c+x)}} .
\end{aligned}
$$

In fact, denoting the $\operatorname{logarithm}$ by $\log \frac{P+Q}{P-Q}$, we have

where

$$
\frac{d}{d x} \log \frac{P+Q}{P-Q}=\frac{2\left(P Q^{\prime}-P^{\prime} Q\right)}{P^{2}-Q^{2}}
$$

$$
\begin{aligned}
2\left(P Q^{\prime}-P^{\prime} Q\right)=2 P Q\left(\frac{Q^{\prime}}{Q}-\frac{P^{\prime}}{P}\right) & =\sqrt{x(a+x)(c+x) b(a-b)(b-c)}\left\{\frac{1}{a+x}+\frac{1}{c+x}-\frac{1}{x}\right\} \\
& =\frac{\sqrt{b(a-b)(b-c)}}{\sqrt{x(a+x)(c+x)}}\left(x^{2}-a c\right) \\
P^{2}-Q^{2} & =-x(a-b)(b-c)+b(a+x)(c+x) \\
& =(b x+a c)(b+x)
\end{aligned}
$$

that is

$$
\begin{aligned}
\frac{2\left(P Q^{\prime}-P^{\prime} Q\right)}{P^{2}-Q^{2}} & =-\frac{\sqrt{b(a-b)(b-c)}}{\sqrt{x(a+x)(c+x)}} \frac{x^{2}-a c}{(b x+a c)(b+x)}, \\
& =\frac{\sqrt{b(a-b)(b-c)}}{\sqrt{x(a+x)(c+x)}}\left\{-\frac{1}{b}+\frac{x}{b(b+x)}+\frac{x}{b x+a c}\right\},
\end{aligned}
$$

which proves the theorem.

19. Hence in the particular case $a c=b^{2}$ we have

$$
\begin{gathered}
\frac{1}{\sqrt{b(a-b)(b-c)}} \log \frac{\sqrt{-h(a-b)(b-c)}+\sqrt{-b(a+h)(c+h)}}{\sqrt{-h(a-b)(b-c)}-\sqrt{-b(a+h)(c+h)}}, \\
=-\frac{1}{b} \int_{-a}^{h} \frac{d h}{\sqrt{h(a+h)(c+h)}} \\
\quad-\frac{2}{b} \int_{-a}^{h} \frac{d h}{b+h} \sqrt{\frac{h}{(a+h)(c+h)}}\left(=+\frac{2}{b} \Pi(h)\right),
\end{gathered}
$$


that is

$$
\Pi(h)=\frac{1}{2} \int_{-a}^{h} \frac{d h}{\sqrt{h(a+h)(c+h)}}+\frac{1}{2} \sqrt{(a-b)(b-c)} \log \frac{\sqrt{-h}(a-b)(b-c)}{\sqrt{-h(a-b)(b-c)}-\sqrt{-b(a+h)(c+h)}},
$$

or say

$$
=\frac{1}{2} \int_{-a}^{h} \frac{d h}{\sqrt{h(a+h)(c+h)}}+\frac{1}{2} \sqrt{\frac{b}{(a-b)(b-c)}} \log \frac{1+H}{1-H}
$$

where

$$
H^{2}=\frac{b}{(a-b)(b-c)} \frac{(a+h)(c+h)}{h},
$$

viz. we see that $\Pi(h)$ depends on the more simple integral

$$
\int_{-a}^{h} \frac{d h}{\sqrt{h(a+h)(c+h)}}
$$

20. Similarly

$$
\begin{aligned}
& \frac{1}{\sqrt{b(a-b)(b-c)}} \log \frac{\sqrt{-k(a-b)(b-c)}+\sqrt{-b(a+k)(c+k)}}{\sqrt{-k(a-b)(b-c)}-\sqrt{-b(a+k)(c+k)}} \\
&= \frac{1}{b} \int_{k}^{-c} \frac{d k}{\sqrt{k(a+k)(c+k)}} \\
&+\frac{2}{b} \int_{k}^{-c} b+\frac{d k}{\frac{k}{(a+k)(c+k)}}\left(=+\frac{2}{b} \Psi(k)\right),
\end{aligned}
$$

that is

$\Psi(k)=-\frac{1}{2} \int_{k}^{-c} \frac{d k}{\sqrt{k(a+k)(c+k)}}+\frac{1}{2} \sqrt{\frac{b}{(a-b)(b-c)}} \operatorname{Iog} \frac{\sqrt{-k(a-b)(b-c)}+\sqrt{-b(a+k)(c+k)}}{\sqrt{-k(a-b)(b-c)}-\sqrt{-b(a+k)(c+k)}}$, or say

$\Psi(k)=-\frac{1}{2} \int_{k}^{-c} \frac{d k}{\sqrt{k(a+k)(c+k)}}+\frac{1}{2} \sqrt{\frac{b}{(a-b)(b-c)} \log \frac{1+K}{1-K}}$,

where

$$
K^{2}=\frac{b}{(a-b)(b-c)} \frac{(a+k)(c+k)}{k}
$$

that is, $\Psi(k)$ depends on the more simple integral,

$$
\int_{k}^{-c} \frac{d k}{\sqrt{k(a+k)(c+k)}}
$$

Write $h=-b-u, k=-b+v$, where $u$ and $v$ are indefinitely small, then

$$
\Pi(h)-\Psi(k)=\frac{1}{2} \int_{-a}^{-c} \frac{d h}{\sqrt{h}(a+h)(c+h)}+\frac{1}{2} \sqrt{\frac{b}{(a-b)(b-c)}} \log \frac{1+U}{1-U} 1-V,
$$


where

$$
U^{2}=\frac{\left(1-\frac{u}{a-b}\right)\left(1+\frac{u}{b-c}\right)}{1+\frac{u}{b}}=1-\frac{b u^{2}}{(a-b)(b-c)(b+u)}\left(\text { attending to } a c=b^{2}\right)
$$

and

$$
\begin{aligned}
& V^{2}= \frac{\left(1+\frac{v}{a-b}\right)\left(1-\frac{v}{b-c}\right)}{1-\frac{v}{b}}=1-\frac{b v^{2}}{(a-b)(b-c)(b-v)}, \\
& \Pi(h)-\Psi(k)=\frac{1}{2} \int_{-a}^{-c} \frac{d h}{\sqrt{h(a+h)(c+h)}}+\sqrt{\frac{b}{(a-b)(b-c)}} \log \frac{v}{u} .
\end{aligned}
$$

21. Comparing with the result obtained for the general case the two agree, if only

$$
\int_{-a}^{-c} \frac{d h}{d} \sqrt{\frac{h}{(a+h)(c+h)}}=\frac{1}{2} \int_{-a}^{-c} \frac{d h}{\sqrt{h(a+h)(c+h)}},
$$

where on the left-hand side the integral has its principal value: a result which must therefore hold good when $a c=b^{2}$.

Calculation of the Umbilicar Geodesics for Ellipsoid $a: b: c=4: 2: 1$.

22. As a specimen of the way in which we may, on a given ellipsoid, calculate the course of a geodesic line, I take the semiaxes to be as $2: \sqrt{2}: 1$, or, for convenience, $a=1000, b=500, c=250$; and, considering the geodesic lines through the umbilicus, I calculate by quadratures the functions

$$
\begin{aligned}
& \Pi(h)=100,000 \int_{-1000}^{h} \frac{-d h}{500+h} \sqrt{\frac{h}{(1000+h)(250+h)}} \\
& \Psi(k)=100,000 \int_{k}^{-250} \frac{d k}{500+k} \sqrt{\frac{k}{(1000+k)(250+k)}}
\end{aligned}
$$

The results do not pretend to minute accuracy: I have not attempted to estimate or correct for any error occasioned by the intervals (10 units) being too large; and there may possibly be accidental errors. 
TABLE I.

\begin{tabular}{|c|c|c|c|c|c|c|c|c|}
\hline$-h=$ & $\Pi^{\prime}$ & $\Pi(h)$ & $-h$ & $\Pi^{\prime}$ & $\Pi(h)$ & $-h$ & $\Pi^{\prime}$ & $\Pi(h)$ \\
\hline 1000 & $\infty$ & 0 & 840 & $27 \cdot 6$ & 6746 & 630 & $51 \cdot 5$ & 13972 \\
\hline 999 & $231 \cdot 4$ & 462 & 830 & $27 \cdot 8$ & 7023 & 620 & $54 \cdot 1$ & 14499 \\
\hline 998 & $164 \cdot 0$ & 659 & 820 & $27 \cdot 9$ & 7301 & 610 & $59 \cdot 2$ & 15066 \\
\hline 997 & $134 \cdot 2$ & 809 & 810 & $28 \cdot 1$ & 7582 & 600 & $65 \cdot 5$ & 15689 \\
\hline 996 & $116 \cdot 5$ & 934 & 800 & $28 \cdot 4$ & 7865 & 590 & $72 \cdot 1$ & 16377 \\
\hline 995 & $104 \cdot 4$ & 1044 & 790 & $28 \cdot 7$ & 8151 & 580 & $80 \cdot 9$ & 17142 \\
\hline 990 & $74 \cdot 6$ & 1492 & 780 & $29 \cdot 2$ & 8440 & 570 & $93 \cdot 0$ & 18011 \\
\hline 980 & $54 \cdot 0$ & 2135 & 770 & $29 \cdot 7$ & 8735 & 560 & $106 \cdot 8$ & 19010 \\
\hline 970 & $45 \cdot 1$ & 2630 & 760 & $30 \cdot 3$ & 9035 & 550 & $127 \cdot 6$ & 20183 \\
\hline 960 & $40 \cdot 1$ & 3056 & 750 & $31 \cdot 0$ & 9341 & 540 & $159 \cdot 1$ & 21616 \\
\hline 950 & $36 \cdot 6$ & 3439 & 740 & $31 \cdot 8$ & 9655 & 530 & $211 \cdot 5$ & 23469 \\
\hline 940 & $34 \cdot 2$ & 3794 & 730 & $32 \cdot 6$ & 9977 & 520 & $316 \cdot 7$ & 26111 \\
\hline 930 & $32 \cdot 5$ & 4127 & 720 & $33 \cdot 6$ & 10308 & 510 & $632 \cdot 7$ & 30858 \\
\hline 920 & $31 \cdot 2$ & 4446 & 710 & $34 \cdot 7$ & 10650 & 505 & $1265 \cdot 0$ & 35602 \\
\hline 910 & $30 \cdot 2$ & 4753 & 700 & $36 \cdot 0$ & 11004 & 504 & $1581 \cdot 2$ & 37014 \\
\hline 900 & $29 \cdot 4$ & 5051 & 690 & $37 \cdot 4$ & 11371 & 503 & $2107 \cdot 7$ & 38834 \\
\hline 890 & $28 \cdot 8$ & 5342 & 680 & $39 \cdot 0$ & 11754 & 502 & $3162 \cdot 3$ & 41398 \\
\hline 880 & $28 \cdot 4$ & 5628 & 670 & $40 \cdot 8$ & 12153 & 501 & $6324 \cdot 1$ & 45792 \\
\hline 870 & $28 \cdot 1$ & 5911 & 660 & $42 \cdot 0$ & 12567 & 500 & $\infty$ & $\infty$ \\
\hline 860 & $27 \cdot 9$ & 6190 & 650 & $45 \cdot 4$ & 13005 & $\theta$ & & \\
\hline 850 & $27 \cdot 8$ & 6469 & 640 & $48 \cdot 2$ & 13473 & & & \\
\hline
\end{tabular}


TABLE II.

\begin{tabular}{|c|c|c|c|c|c|c|c|c|}
\hline$-k$ & $\Psi^{\prime}$ & $\Psi(k)$ & $-k$ & $\Psi^{\prime}$ & $\Psi(k)$ & $-k$ & $\Psi^{\prime}$ & $\Psi(k)$ \\
\hline 250 & $\infty$. & 0 & 320 & $45 \cdot 5$ & 4655 & 440 & $107 \cdot 1$ & 12207 \\
\hline 251 & $232 \cdot 2$ & 462 & 330 & $46 \cdot 1$ & 5114 & 450 & $127 \cdot 9$ & 13383 \\
\hline 252 & $165 \cdot 5$ & 661 & 340 & $47 \cdot 3$ & 5581 & 460 & $159 \cdot 2$ & 14818 \\
\hline 253 & $136 \cdot 0$ & 811 & 350 & $48 \cdot 9$ & 6062 & 470 & $211 \cdot 6$ & 16673 \\
\hline 254 & $118 \cdot 6$ & 939 & 360 & $51 \cdot 1$ & 6562 & 480 & $316 \cdot 7$ & 19314 \\
\hline 255 & $106 \cdot 8$ & 1051 & 370 & 53.8 & 7086 & 490 & $632 \cdot 7$ & 24062 \\
\hline 260 & $78 \cdot 1$ & 1514 & 380 & $57 \cdot 2$ & 7641 & 495 & $1265 \cdot 0$ & 28806 \\
\hline 270 & $60 \cdot 5$ & 2207 & 390 & $61 \cdot 4$ & 8235 & 496 & $1581 \cdot 2$ & 30218 \\
\hline 280 & $51 \cdot 7$ & 2768 & 400 & $66 \cdot 7$ & 8875 & 497 & $2108 \cdot 1$ & 32037 \\
\hline 290 & $48 \cdot 2$ & 3268 & 410 & $73 \cdot 2$ & 9575 & 498 & $3162 \cdot 3$ & 34602 \\
\hline 300 & $46 \cdot 3$ & 3741 & 420 & $81 \cdot 6$ & 10349 & 499 & $6234 \cdot 1$ & 38995 \\
\hline 310 & $45 \cdot 5$ & 4200 & 430 & $91 \cdot 4$ & 11214 & 500 & $\infty$ & $\infty$ \\
\hline
\end{tabular}

23. But it is obviously convenient to revert these Tables so as to have for the common arguments a series of uniformly increasing values of $\Pi$ or $\Psi$, viz., we obtain by interpolation the values of $h$ and $k$ belonging to the given values of $\Pi$ or $\Psi$, and thus obtain the following Table. Here, in any line of the Table the values of $h, k$ are such that $\Pi(h)-\Psi(k)=0$, viz., the values in question belong to successive points of the $B$-geodesic. And to obtain the values for any other geodesic line $\Pi(h)-\Psi(k)= \pm 500 m$, we have only to take each value of $k$ from the line $m$ lines above or below the line from which $h$ is taken; and similarly the table gives at once the values belonging to a geodesic line $\Pi(h)+\Psi(k)=500 \mathrm{~m}$. 
Table III.

\begin{tabular}{|c|c|c|c|c|c|c|c|c|c|}
\hline$\Pi=\Psi=$ & $h$ & D. & $k$ & D. & $\Pi=\Psi=$ & $h$ & D. & $k$ & D. \\
\hline 0 & 1000 & & 250 & & 13000 & $650 \cdot 1$ & & $446 \cdot 7$ & \\
\hline 500 & $998 \cdot 8$ & & $251 \cdot 4$ & $1 . t$ & 14000 & $629 \cdot 5$ & $20 \cdot 6$ & $454 \cdot 5$ & 1.8 \\
\hline 1000 & $995 \cdot 4$ & 57 & $254 \cdot 5$ & 31 & 15000 & $611 \cdot 2$ & $18 \cdot 3$ & $461 \cdot 0$ & 6.5 \\
\hline 1500 & $989 \cdot 9$ & 5 & $259 \cdot 7$ & 5 & 16000 & $595 \cdot 5$ & $15 \cdot 7$ & $466 \cdot 3$ & $5 \cdot 3$ \\
\hline 2000 & $982 \cdot 1$ & 0. & $267 \cdot 0$ & & 17000 & $581 \cdot 9$ & $13 \cdot 6$ & $471 \cdot 2$ & $4 \cdot 9$ \\
\hline 2500 & $972 \cdot 6$ & & $275 \cdot 2$ & & 18000 & $570 \cdot 1$ & $11 \cdot 8$ & $475 \cdot 0$ & $3 \cdot 8$ \\
\hline 3000 & $961 \cdot 1$ & & $284 \cdot 6$ & & 19000 & $560 \cdot 1$ & $10 \cdot 0$ & 4788 & $3 \cdot 8$ \\
\hline 3500 & $948 \cdot 3$ & & 294.9 & & 20000 & $551 \cdot 6$ & $8 \cdot 5$ & $481 \cdot 4$ & $2 \cdot 6$ \\
\hline 4000 & $933 \cdot 8$ & & $305 \cdot 6$ & & 21000 & $544 \cdot 3$ & $7 \cdot 3$ & $483 \cdot 6$ & $2 \cdot 2$ \\
\hline 4500 & $918 \cdot 2$ & & $316 \cdot 6$ & & 22000 & $538 \cdot 1$ & $6 \cdot 2$ & $485 \cdot 6$ & $2 \cdot 0$ \\
\hline 5000 & $901 \cdot 7$ & & $327 \cdot 5$ & 0.8 & 23000 & $533 \cdot 2$ & $4 \cdot 9$ & $487 \cdot 8$ & $2 \cdot 2$ \\
\hline 5500 & $884 \cdot 5$ & & $338 \cdot 3$ & & 24000 & $528 \cdot 6$ & & $489 \cdot 9$ & $2 \cdot 1$ \\
\hline 6000 & $866 \cdot 8$ & .9 & $348 \cdot 7$ & $10 \cdot 1$ & 26000 & $520 \cdot 5$ & $8 \cdot 1$ & $492 \cdot 0$ & \\
\hline 6500 & $848 \cdot 9$ & & $358 \cdot 8$ & & 28000 & $516 \cdot 0$ & 4.5 & $494 \cdot 1$ & $2 \cdot 1$ \\
\hline 7000 & $830 \cdot 8$ & & $368 \cdot 3$ & $9 \cdot 2$ & 30000 & $511 \cdot 8$ & $4 \cdot 2$ & $495 \cdot 8$ & 8 \\
\hline 7500 & $812 \cdot 9$ & $17 \cdot 6$ & $377 \cdot 5$ & $8 \cdot 6$ & 32000 & $508 \cdot 8$ & $3 \cdot($ & $497 \cdot 0$ & \\
\hline 8000 & $795 \cdot 3$ & $17 \cdot 3$ & $386 \cdot 1$ & $8 \cdot 0$ & 34000 & $506 \cdot 7$ & & $497 \cdot 8$ & 5 \\
\hline 8500 & $778 \cdot 0$ & $16 \cdot 8$ & $394 \cdot 1$ & $7 \cdot 7$ & 36000 & $504 \cdot 7$ & & $498 \cdot 3$ & \\
\hline 9000 & $761 \cdot 2$ & 16 & $401 \cdot 8$ & & 38000 & 503.5 & & $498 \cdot 8$ & \\
\hline 9500 & $744 \cdot 9$ & $15 \cdot 6$ & $408 \cdot 9$ & $6 \cdot 6$ & 39000 & & & $499 \cdot 0$ & \\
\hline 10000 & $729 \cdot 3$ & $14 \cdot 9$ & $415 \cdot 5$ & 6. & 40000 & $502 \cdot 5$ & & 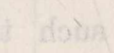 & \\
\hline 10500 & $714 \cdot 4$ & $14 \cdot 3$ & $421 \cdot 7$ & 5 & 42000 & $501 \cdot 9$ & 0 & & \\
\hline 11000 & $700 \cdot 1$ & 13.5 & $427 \cdot 6$ & & 44000 & $501 \cdot 4$ & & & \\
\hline 11500 & $686 \cdot 6$ & $12 \cdot 8$ & $432 \cdot 9$ & $5 \cdot 0$ & 45800 & $501 \cdot 0$ & $0 \cdot 4$ & & \\
\hline 12000 & $673 \cdot 8$ & $23 \cdot 7$ & $437 \cdot 9$ & $8 \cdot 8$ & $\infty$ & 500 & & 500 & \\
\hline
\end{tabular}




\section{Graphical Construction: Projection on the Umbilicar Plane.}

24. The most convenient mode of delineation of the geodesic lines is obtained by projecting them orthogonally on the umbilicar plane: the contour of the figure is here the umbilicar section, or ellipse $\frac{x^{2}}{a}+\frac{z^{2}}{c}=1$; and the curves of curvature of each series are projected into elliptic arcs lying within the ellipse in question, the one set cutting at right angles the axes $A A^{\prime}$, the other cutting at right angles the axes $C C^{\prime}$; the equations of the complete ellipses being

and

$$
x^{2} \frac{a-b}{a(a+h)}+z^{2} \frac{c-b}{c(c+h)}-1=0
$$

$$
x^{3} \frac{a-b}{a(a+k)}+z^{2} \frac{c-b}{c(c+k)}-1=0 .
$$

25. I constructed, by means of the table, a drawing of this kind for the ellipsoid $a, b, c=1000,500,250$, the lengths $\sqrt{a}$ and $\sqrt{c}$ being taken to be 12 inches and 6 inches respectively: the process consists in taking from the table for a series of values $\Pi=\Psi$ (say $\Pi=\Psi=1000,=2000 \&$ c), the values of $h$ and $k$, laying down for such values the elliptic arcs which represent the two curves of curvature respectively, thus dividing the bounding ellipse into a series of curvilinear rectangles, and then obtaining the geodesic lines by drawing the diagonals of these rectangles, and of course rounding off the corners so as to form continuous curves. The Plate shows on a reduced scale so much of the drawing as is comprised within a quadrant of the bounding ellipse (viz. it is a representation of an octant of the ellipsoid).

\section{Elliptic-Function Formulce.}

26. I have in all that precedes abstained from the use of elliptic functions, since obviously the form $\sqrt{1-k^{2} \sin ^{2} \phi}$ of the radical of an elliptic function is in nowise specially appropriate to the present question. But (more particularly in the abovementioned case $a c-b^{2}=0$, where the radical is $\sqrt{ } h(a+h)(c+h)$ without any exterior factor $b+h$ in the denominator) the formulæ are expressible easily and elegantly by elliptic functions; and it is desirable to make the transformation. Reverting to the formulæ which, in the case in question (viz. when $a c-b^{2}=0$ ), give the values of $\Pi(h)$ and $\Psi(k)$; and writing therein $h=-a+(a-c) \sin ^{2} \phi, k=-a+(a-c) \sin ^{2} \psi$, also

we have

$$
\kappa=\sqrt{1-\frac{c}{a}}, \text { or } \frac{c}{a}=1-\kappa^{2},=\kappa^{\prime 2},
$$

$$
\begin{aligned}
& \int_{-a}^{h} \frac{d h}{\sqrt{h(a+h)(c+h)}}=2 \int_{0}^{\phi} \frac{d \phi}{\sqrt{a-(a-c) \sin ^{2} \phi}}=\frac{2}{\sqrt{a}} F(\kappa, \phi), \\
& \int_{k}^{-c} \frac{d k}{\sqrt{k(a+k)(c+k)}}=2 \int_{\psi}^{\frac{1}{2} \pi} \frac{d \psi}{\sqrt{a-(a-c) \sin ^{2} \psi}}=\frac{2}{\sqrt{a}}\{F(\kappa)-F(\kappa, \psi)\} .
\end{aligned}
$$


27. Hence

$$
\Pi(h)=\frac{1}{\sqrt{a}} F(\kappa, \phi)+\frac{1}{2 \sqrt{a\left(1-\kappa^{\prime}\right)}} \log \frac{1+H}{1-H},
$$

where

$$
H=\frac{\left(1-\kappa^{\prime}\right) \sin \phi \cos \phi}{\sqrt{1-\kappa^{2} \sin ^{2} \phi}}
$$

(observe, as $h$ passes from $-a$ to $-b, \phi$ passes from $\phi=0$ to $\sin ^{2} \phi=\frac{1}{1+\kappa}$ and $H$ from $H=0$ to $H=1$ ).

Similarly

$$
\Psi(k)=\frac{1}{\sqrt{a}}\left\{F^{\prime},(\kappa)-F^{\prime}(\kappa, \psi)\right\}+\frac{1}{2 \sqrt{a\left(1-\kappa^{\prime}\right)}} \log \frac{1+K}{1-K}
$$

where

$$
K=\frac{\left(1+\kappa^{\prime}\right) \sin \psi \cos \psi}{\sqrt{1-\kappa^{2} \sin ^{2} \psi}}
$$

and as $k$ passes from $-c$ to $-b, \psi$ passes from $\frac{1}{2} \pi$ to $\sin ^{2} \psi=\frac{1}{1+\kappa}$, and $K$ from 0 to 1 .

28. The before-mentioned identical equation

$$
\int_{-a}^{-c} \frac{d h}{b+h} \sqrt{\frac{h}{(a+h)(c+h)}}=\frac{1}{2} \int_{-a}^{-c} \frac{d h}{\sqrt{h(a+h)(c+h)}}
$$

is by the same transformation converted into

$$
\int_{0}^{\frac{1}{2} \pi} \frac{1-\left(1-\kappa^{\prime}\right) \sin ^{2} \phi}{1-\left(1+\kappa^{\prime}\right) \sin ^{2} \phi} \frac{d \phi}{\sqrt{1-\kappa^{2} \sin ^{2} \phi}}=0 .
$$

To prove this, I remark that the equation is

viz. this is

$$
0=\int_{-0}^{\frac{1}{2} \pi} d \phi \frac{\frac{1-\kappa^{\prime}}{1+\kappa^{\prime}}\left\{1-\left(1+\kappa^{\prime}\right) \sin ^{2} \phi\right\}+\frac{2 \kappa^{\prime}}{1+\kappa^{\prime}}}{1-\left(1+\kappa^{\prime}\right) \sin ^{2} \phi} \frac{1}{\Delta \phi}
$$

$$
0=\frac{1-\kappa^{\prime}}{1+\kappa^{\prime}} F^{\prime}+\frac{2 \kappa}{1+\kappa^{\prime}} \Pi,\left(-1-\kappa^{\prime}\right)
$$

or, what is the same thing,

$$
\Pi,\left(-1-\kappa^{\prime}\right)=-\frac{1-\kappa^{\prime}}{2 \kappa} F_{,}
$$

where $\Pi,\left(-1-\kappa^{\prime}\right)$ denotes the principal value of the integral

$$
\int_{0}^{\frac{1}{2} \pi} d \phi \frac{1}{1-\left(1+\kappa^{\prime}\right) \sin ^{2} \phi} \frac{1}{\Delta \phi} .
$$


Now (Leg. Fonct. Ellip., t. I., p. 71), we have

$$
\Pi_{,}\left(-\kappa^{2} \sin ^{2} \theta\right)+\Pi_{c}\left(-\frac{1}{\sin ^{2} \theta}\right)=F_{c}
$$

where, upon examination, it will appear that $\Pi_{,}\left(-\frac{1}{\sin ^{2} \theta}\right)$ in fact represents the principal value of the integral.

Writing herein $\sin ^{2} \theta=\frac{1}{1-\kappa^{\prime}}$, and therefore $\cos ^{2} \theta=\frac{\kappa^{\prime}}{1+\kappa^{\prime}}$, or $\tan ^{2} \theta=\kappa^{\prime}$, this is

$$
\Pi_{,}\left(-1+\kappa^{\prime}\right)+\Pi_{,}\left(-1-\kappa^{\prime}\right),=F_{\prime},
$$

and the formula $\left(p^{\prime}\right)$, p. 141, attributing therein to $\theta$ the foregoing value, becomes

$$
\Pi,\left(-1-\kappa^{\prime}\right)=E,+\frac{1}{\kappa^{\prime}}\left\{F, E(\theta)-E, F^{\prime}(\theta)\right\} .
$$

But $\theta$ is the value for the bisection of the function $F_{1}$, viz., we have

whence

$$
\begin{aligned}
& 2 F(\theta)=F, \\
& 2 E(\theta)=E,+1-\kappa^{\prime},
\end{aligned}
$$

$$
F, E(\theta)-E, F(\theta)=\frac{1}{2}\left(1-\kappa^{\prime}\right) F_{,},
$$

or the formula in question gives

whence

$$
\Pi,\left(-1+\kappa^{\prime}\right)=\frac{1+\kappa^{\prime}}{2 \kappa^{\prime}} E_{\iota},
$$

the result which was to be proved.

$$
\Pi,\left(-1-\kappa^{\prime}\right)=-\frac{1-\kappa^{\prime}}{2 \kappa^{\prime}} F_{\prime}^{\prime}
$$

29. The value of $M^{\prime}$ (observing that $\left.\frac{b}{(a-b)(b-c)}=\frac{1}{(\sqrt{a}-\sqrt{c})^{2}}=\frac{1}{a\left(1-\kappa^{\prime}\right)^{2}}\right)$ is

which is

$$
\frac{1}{\sqrt{a}\left(1-\kappa^{\prime}\right)} M^{\prime}=\frac{1}{2} \int_{-a}^{-c} \sqrt{h(a+h)(c+h)},
$$

that is we have

$$
=\frac{1}{2} \frac{2}{\sqrt{2}} F,(\kappa) \text {, }
$$

or, what is the same thing,

$$
M^{\prime}=\left(1-\kappa^{\prime}\right) F^{\prime}(\kappa)
$$

that is

$$
\log \tan \frac{1}{2} \phi_{0}=\frac{1-\kappa^{\prime}}{2} F_{,},(\kappa)
$$

$$
\tan \frac{1}{2} \phi_{0}=\left(\frac{1-\kappa^{\prime}}{2} F_{,}(\kappa)\right)
$$

( $\phi_{0}$ the South azimuth of the $B$-geodesic at the umbilicus).

30. I purposely calculated the Table by quadratures as being a method available where the equation $a c-b^{2}=0$ is not satisfied; but in the present case, where this 
equation is satisfied, the table might have been calculated from Legendre's Tables of Elliptic Integrals. Observe that $a=1000, b=500, c=250$, gives $\kappa=\frac{1}{2} \sqrt{3}$ or angle of modulus $=60^{\circ}$. As an instance of the comparison $\left({ }^{1}\right)$, suppose $h=-800$, then $\sin ^{2} \phi=\frac{200}{75}=\frac{4}{15}$, $\log \sin \phi=9 \cdot 71298, \phi=31^{\circ} 5^{\prime}$.

$$
\begin{aligned}
& \sqrt{\frac{b}{(a-b)(b-c)}}=\sqrt{\frac{500}{500.250}}=\frac{\sqrt{10}}{50}=06326, \\
& H^{2}=\frac{500 \cdot 200.550}{800.500 \cdot 250}=\frac{110}{200}, \log =\overline{1} \cdot 87018, \\
& H=\cdot 7416 \frac{1+H}{1-H}=\frac{1 \cdot 7416}{2 \cdot 584}=6 \cdot 7582, \\
& \Pi(h)=\cdot 03163 F^{\prime}\left(31^{\circ} 5^{\prime}\right)+\cdot 03163 \text { h. 1. } 6 \cdot 7582, \\
& F 31^{\circ}=56166 \\
& 163 \\
& F 31^{\circ} 5^{\prime}=\overline{.56329} \\
& \text { h. } 1.6 \cdot 7582=\frac{1.91075}{2.47404} \\
& \times \text { by } \quad 03163 \\
& .0782043
\end{aligned}
$$

or multiplying by 100,000 (factor introduced into my Table) this is $=7820.43$. The value $\Pi(-800)=7864$ given by my Table agrees sufficiently well with this, the correct value.

31. I calculate also the angle $\phi_{0}$, viz. we have

$$
\begin{aligned}
\text { h. l. } \tan \frac{1}{2} \phi_{0}=\frac{1-\kappa^{\prime}}{2} F_{, \kappa,} & =\frac{1}{4} F^{\prime},\left(60^{\circ}\right) . \quad \text { Leg. vol. III. Table viII. } \\
& =\frac{1}{4} 2 \cdot 15651=53913,
\end{aligned}
$$

whence by Leg. Table Iv.

$$
\begin{aligned}
\frac{1}{2} \phi_{0} & =45^{\circ}+\frac{1}{2} \cdot 29^{\circ} 29^{\prime} \cdot 64 \\
& =59^{\circ} 44^{\prime} \cdot 82
\end{aligned}
$$

or

$$
\phi_{0}=119^{\circ} 29^{\prime} \cdot 64 \text {. }
$$

This exceeds $90^{\circ}$, and since at the umbilicus the tangent plane is at right angles to the plane of projection, the $B$-geodesic should in the drawing proceed (as it in fact does) from $U$ in the sense $U C$, touching the bounding ellipse at the point $U$. as h. 1 .

1 In the present calculation, log denotes an ordinary logarithm, the hyperbolic logarithm being distinguished 


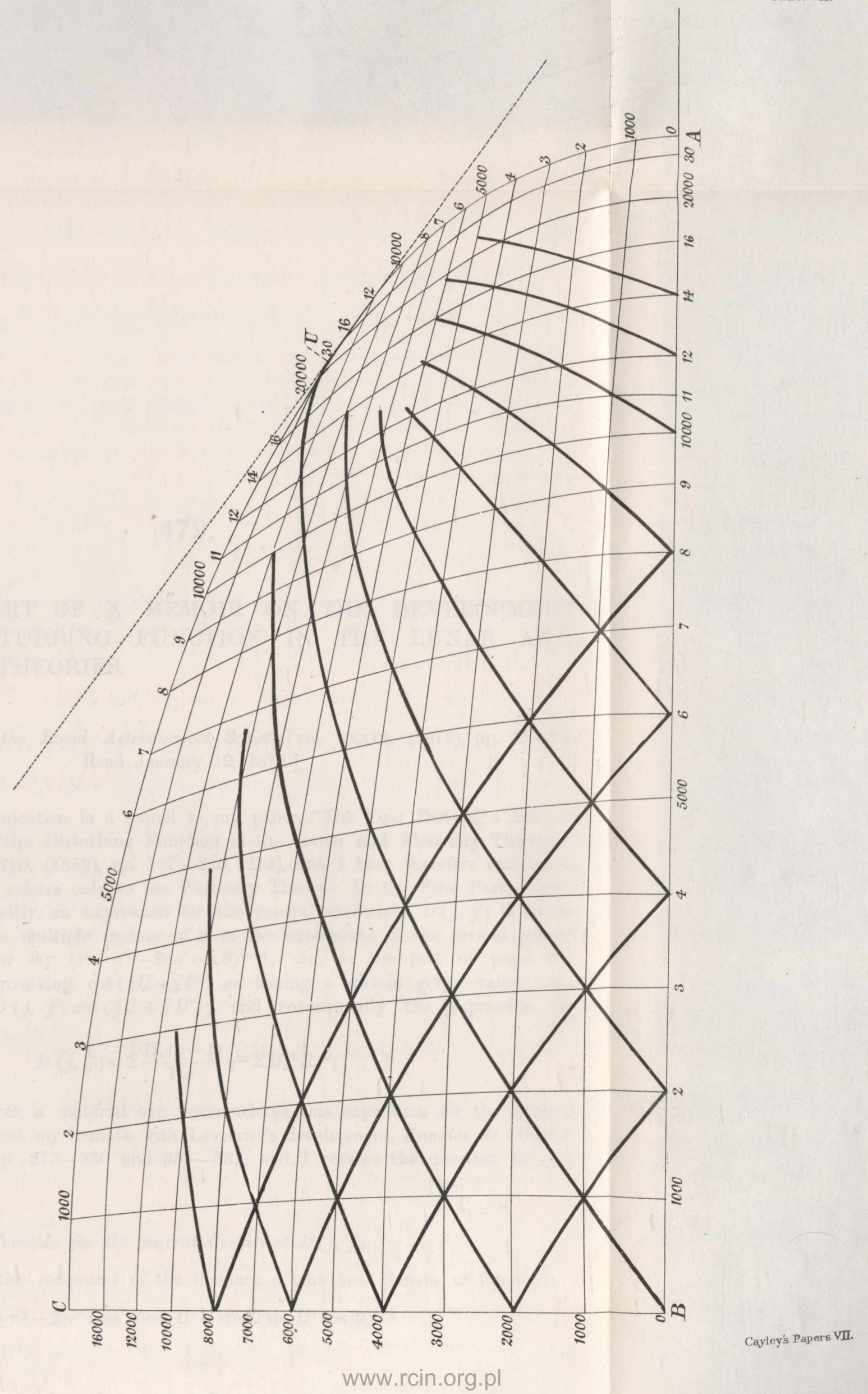

